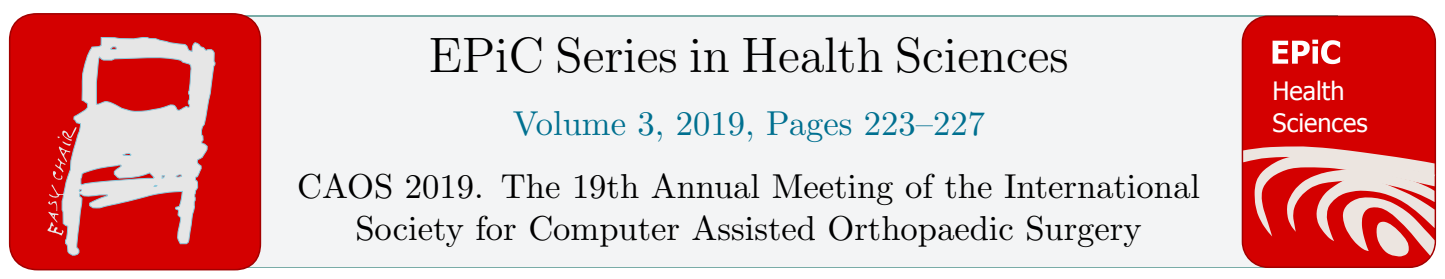

\title{
Peak Active Hip Flexion Following Navigated Total Hip Arthroplasty.
}

\author{
Hollie J. Leonard, Alistair M. Ewen and Kamal Deep \\ Golden Jubilee National Hospital,Clydebank, Scotland, U.K. \\ Hollie.leonard@gjnh.scot.nhs.uk
}

\begin{abstract}
The importance of accurate cup positioning during total hip arthroplasty (THA) to minimise postoperative complications has been well defined. However it remains unclear if following navigated THA there is a relationship between the active range of movement, the inclination and anteversion of the implant, and the theoretical range of movement as measured intra-operatively using imageless navigation. Fifteen male patients undergoing primary THA using the Orthopilot (Aesculap AG, Tuttlingen, Germany) imageless navigation system were recruited. The Orthopilot system flexion value was recorded. Three months post-THA patients underwent 3D biomechanical analysis (Vicon Motion Systems, Oxford, UK) during which patients performed a standing active hip flexion movement and a sitting task. Inclination and anteversion calculated according to Pradhan's formula were taken from post-operative radiographs. There was no observed correlation with the theoretical hip flexion and the standing peak active or sitting peak hip flexion values in this series. However, Orthopilot flexion considers only the interaction of the implant components and not the soft tissues surrounding the hip joint which may limit clinical flexion. There does not appear to be any relationship between the inclination and anteversion angles and the range of movement observed postoperatively during a standing active hip flexion task and sitting task. It was observed that peak active hip flexion between the operated and non-operated limbs was not significantly different at three months, which indicates a good level of symmetry at this time point.
\end{abstract}

\section{Introduction}

It has been well established that malpositioning of the cup during total hip arthroplasty (THA) is associated with dislocation, impingement, accelerated wear, component loosening and migration. Lewinnek at al. (Lewinnek, Lewis, Tarr, Compere, \& Zimmerman, 1978) discussed the importance of cup orientation and defined a safe zone for inclination $\left(40^{\circ}+/-10^{\circ}\right)$ and anteversion $\left(15^{\circ}+/-10^{\circ}\right)$ to minimise post-operative complications. Several studies have reported imageless navigation as having more consistent cup placement than manual techniques(Kelley \& Swank, 2009; Lass et al., 2014). Hohman et al(Hohmann, Bryant, \& Tetsworth, 2011) reported a significant increase in the accuracy of the acetabular cup placement within the desired position and safe zones when using navigation compared to manual freehand technique. In more recent years, there has been an increase in studies examining post-operative function following joint arthroplasty and there are numerous reports on 
post-operative range of movement following THA(Sugano et al., 2012). However it remains unclear if following navigated THA there is a relationship between the active range of movement, the inclination and anteversion of the implant, and the theoretical range of movement as measured intraoperatively using imageless navigation.

\section{Methods}

Fifteen male patients undergoing primary THA using the Excia-T stem (Aesculap AG, Tuttlingen, Germany) and Orthopilot (Aesculap AG, Tuttlingen, Germany) imageless navigation system for osteoarthritis of the hip were recruited. The Orthopilot system displays the virtual range of movements expected from orientation of the acetabular cup and femoral stem. The hip flexion value from the navigation system was recorded.

Three months post-THA patients underwent 3D biomechanical analysis using a 10 camera motion analysis system (Vicon Motion Systems, Oxford, UK), sampling at $100 \mathrm{HZ}$. The Full Body Plug-in Gait marker set (Vicon Motion Systems, Oxford, UK) was applied to all subjects, with two additional markers placed half-way between the anterior superior iliac spine and posterior superior iliac spine on the left and right side of the pelvis. The thigh and tibia markers were placed on stalks. All remaining markers were placed per the Plug-in marker set standard. Patients performed a standing active hip flexion movement in the capture volume, using the support of two height-adjusted walking sticks for support (Figure 1). Patients were then asked to sit down upon, and then rise from, a stool which was height adjusted to the level of the knee joint line(Figure 2). The sitting task was repeated three times, and biomechanical data from the three trials averaged. Data processing and biomechanical outputs were performed in Vicon Nexus (ver. 2.8).

Inclination and anteversion calculated according to Pradhan's formula(Pradhan, 1999) were taken from post-operative radiographs prior to hospital discharge.

Statistical differences were calculated with Paired T-tests and a Pearson product-moment correlation was used to determine the relationship between variables. 


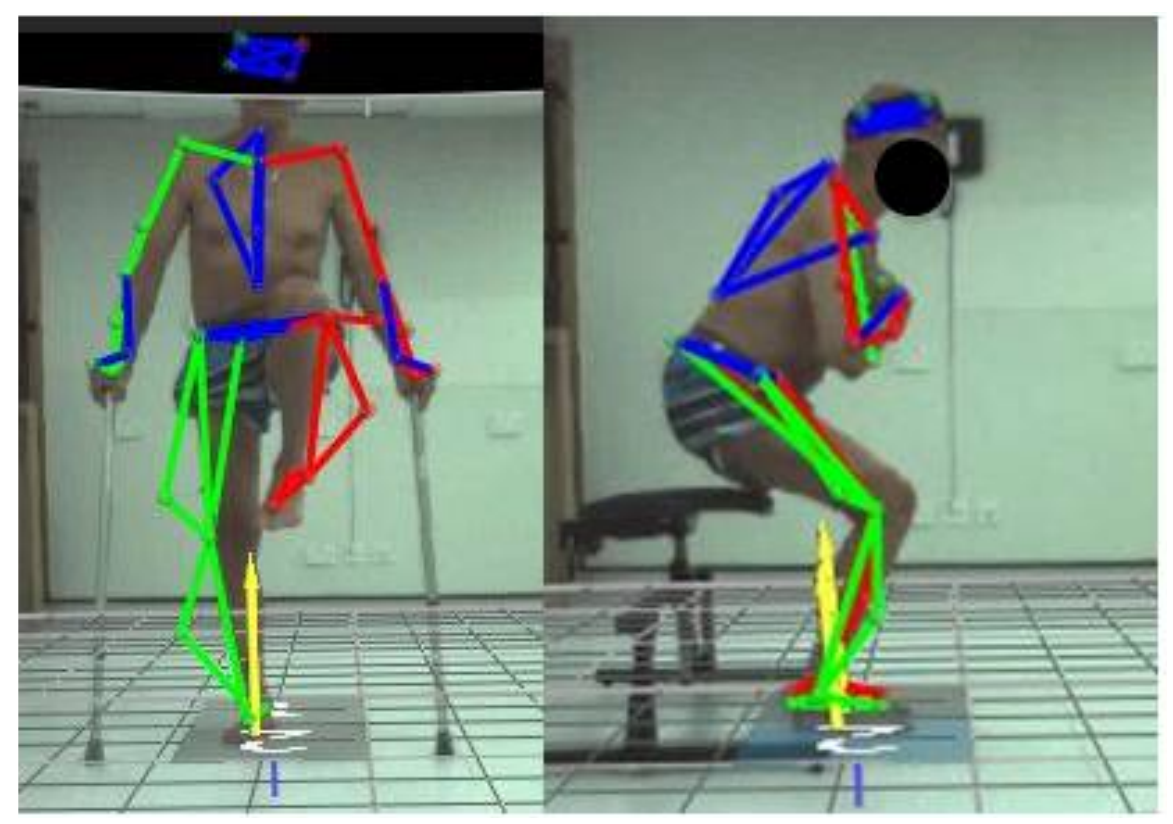

Figure 1: Standing hip flexion task and stand-to-sit-to-stand task.

\section{Results}

The mean age of the cohort was 71.3 years (SD 6.22years) with a mean height of $1.72 \mathrm{~m}$ (SD $0.07 \mathrm{~m}$ ), weight of $83.08 \mathrm{~kg}$ (SD 16.33kg) and BMI of 28.04 (SD 5.35). The theoretical hip flexion recorded by Orthopilot was $117.33^{\circ}$ (SD $7.27^{\circ}$ ), the standing peak hip flexion at three months postTHA was $85.14^{\circ}$ (SD $7.94^{\circ}$ ) and peak hip flexion during the sitting task was $86.93^{\circ}$ (SD $4.90^{\circ}$ ) (Figure 1). There was no relationship between the Orthopilot flexion and the standing active hip flexion $(\mathrm{r}=0.501, \mathrm{p}=0.057)$, or the sitting peak flexion $(\mathrm{r}=0.413, \mathrm{p}=0.126)$. The mean inclination was $39.45^{\circ}\left(\mathrm{SD} 2.82^{\circ}\right)$ and anteversion was $11.30^{\circ}\left(\mathrm{SD} 1.67^{\circ}\right)$, and there was no relationship between the radiographic findings and the hip flexion values. There was a moderate positive relationship between the standing peak active flexion and peak flexion during the sitting task $(\mathrm{r}=0.645, \mathrm{p}=0.009)$. There was no significant difference between the operated $\left(85.14^{\circ}, \mathrm{SD} 7.94^{\circ}\right)$ and non-operated $\left(84.32^{\circ}, \mathrm{SD}\right.$ $\left.7.95^{\circ}\right)$ limbs in standing active flexion $(\mathrm{p}=.720)$. 


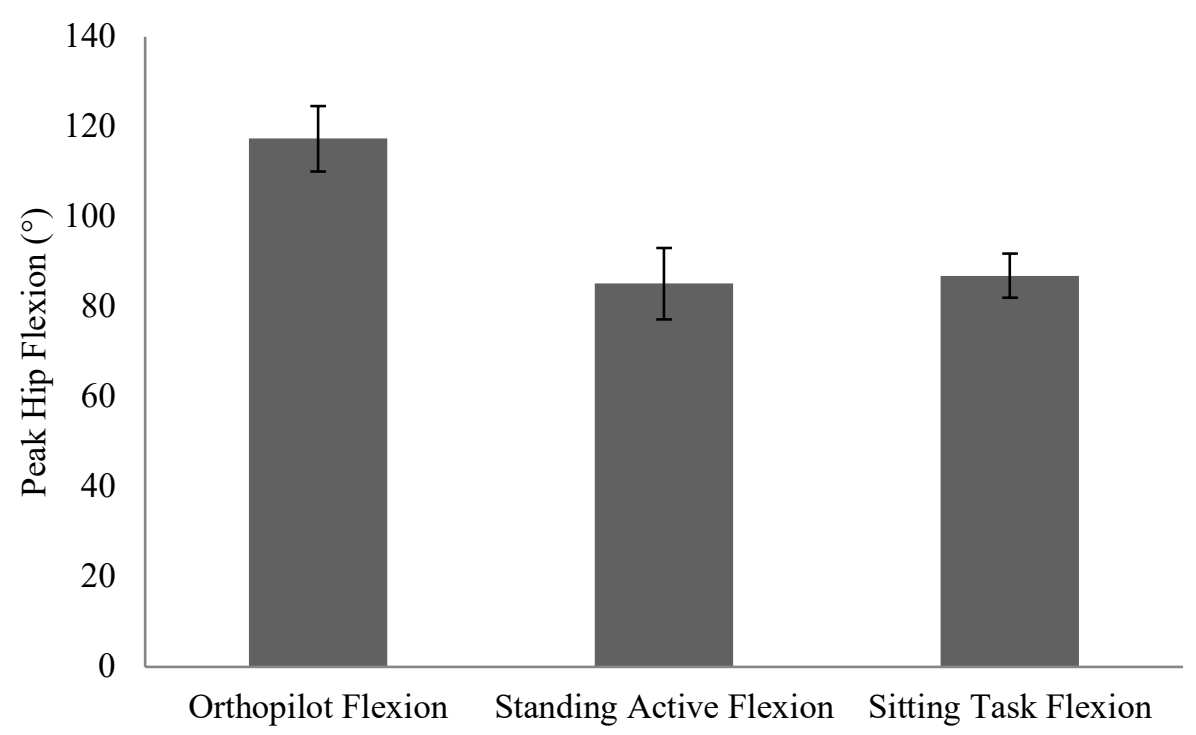

Figure 2. Peak hip flexion values (degrees) recorded by Orthopilot navigation system and 3D biomechanical analysis of functional tasks.

\section{Discussion}

There was no observed correlation with the theoretical hip flexion and the standing peak active or sitting peak hip flexion values in this series. However the Orthopilot flexion considers only the interaction of the implant components and not the soft tissues surrounding the hip joint which could limit the clinical flexion observed. There does not appear to be any relationship between the inclination and anteversion angles and the range of movement observed post-operatively during a standing active hip flexion task and sitting task following navigated THA. The peak hip flexion values reported are in line with those described in the literature(Lamontagne, Beaulieu, Varin, \& Beaulé, 2012; Sugano et al., 2012). It was observed that peak active hip flexion between the operated and nonoperated limbs was not significantly different at three months, which indicates a good level of symmetry at this time point. This is in contrast to the findings of Lamontagne et al(Lamontagne et al., 2012) who report asymmetries in peak hip flexion in the operated and non-operated hips during a sitting task at a minimum follow up time of 6 months after non-navigated THA. While there was statistically greater peak flexion in the sitting task compared to the standing task, it was not a clinically significant result with the mean difference between the tasks being less than two degrees. This series comprises the early results of a larger clinical trial and further analysis will include the effect of BMI and limb dominance on functional range of movement.

In summary, despite there being no apparent correlation to the radiographic findings and functional sagittal plane movement in this cohort, we did observe that navigated THA produced equivalent hip flexion to the contralateral side in the standing active hip flexion task. 


\section{References}

Hohmann, E., Bryant, A., \& Tetsworth, K. (2011). A comparison between imageless navigated and manual freehand technique acetabular cup placement in total hip arthroplasty. The Journal of Arthroplasty, 26(7), 1078-1082.

Kelley, T. C., \& Swank, M. L. (2009). Role of navigation in total hip arthroplasty. JBJS, 91(Supplement 1), 153-158.

Lamontagne, M., Beaulieu, M. L., Varin, D., \& Beaulé, P. E. (2012). Lower-limb joint mechanics after total hip arthroplasty during sitting and standing tasks. Journal of Orthopaedic Research, 30(10), 1611-1617.

Lass, R., Kubista, B., Olischar, B., Frantal, S., Windhager, R., \& Giurea, A. (2014). Total hip arthroplasty using imageless computer-assisted hip navigation: A prospective randomized study. The Journal of Arthroplasty, 29(4), 786-791.

Lewinnek, G. E., Lewis, J. L., Tarr, R., Compere, C. L., \& Zimmerman, J. R. (1978). Dislocations after total hip-replacement arthroplasties. The Journal of Bone and Joint Surgery.American Volume, 60(2), 217-220.

Pradhan, R. (1999). Planar anteversion of the acetabular cup as determined from plain anteroposterior radiographs. The Journal of Bone and Joint Surgery. British Volume, 81(3), 431-435.

Sugano, N., Tsuda, K., Miki, H., Takao, M., Suzuki, N., \& Nakamura, N. (2012). Dynamic measurements of hip movement in deep bending activities after total hip arthroplasty using a 4dimensional motion analysis system. The Journal of Arthroplasty, 27(8), 1562-1568. 